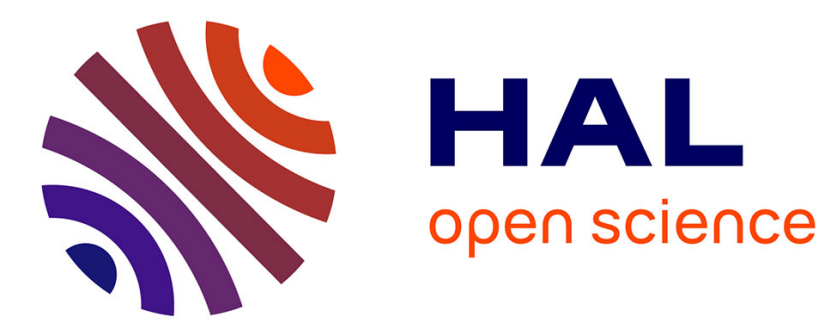

\title{
Unveiling Extreme Anisotropy in Elastic Structured Media
}

G. Lefebvre, T. Antonakakis, Y. Achaoui, R. v. Craster, Sebastien Guenneau, P. Sebbah

\section{- To cite this version:}

G. Lefebvre, T. Antonakakis, Y. Achaoui, R.v. Craster, Sebastien Guenneau, et al.. Unveiling Extreme Anisotropy in Elastic Structured Media. Physical Review Letters, 2017, 118 (25), pp.254302. 10.1103/PhysRevLett.118.254302 . hal-01637653

\section{HAL Id: hal-01637653 https://hal.science/hal-01637653}

Submitted on 12 Apr 2018

HAL is a multi-disciplinary open access archive for the deposit and dissemination of scientific research documents, whether they are published or not. The documents may come from teaching and research institutions in France or abroad, or from public or private research centers.
L'archive ouverte pluridisciplinaire HAL, est destinée au dépôt et à la diffusion de documents scientifiques de niveau recherche, publiés ou non, émanant des établissements d'enseignement et de recherche français ou étrangers, des laboratoires publics ou privés. 


\title{
Unveiling Extreme Anisotropy in Elastic Structured Media
}

\author{
G. Lefebvre, ${ }^{1}$ T. Antonakakis, ${ }^{2}$ Y. Achaoui, ${ }^{3}$ R. V. Craster, ${ }^{4}$ S. Guenneau, ${ }^{3}$ and P. Sebbah ${ }^{1,5, *}$ \\ ${ }^{1}$ Institut Langevin, ESPCI ParisTech CNRS UMR7587, 1 rue Jussieu, 75238 Paris cedex 05, France \\ ${ }^{2}$ Multiwave Technologies AG, 3 Chemin du Pré Fleuri,1228 Geneva, Switzerland \\ ${ }^{3}$ Aix-Marseille Université, CNRS, Centrale Marseille, Institut Fresnel, 13013 Marseille, France \\ ${ }^{4}$ Department of Mathematics, Imperial College London, London SW7 2AZ, United Kingdom \\ ${ }^{5}$ Department of Physics, The Jack and Pearl Resnick Institute for Advanced Technology, Bar-Ilan University, \\ Ramat-Gan 5290002, Israel
}

(Received 7 November 2016; published 20 June 2017)

\begin{abstract}
Periodic structures can be engineered to exhibit unique properties observed at symmetry points, such as zero group velocity, Dirac cones, and saddle points; identifying these and the nature of the associated modes from a direct reading of the dispersion surfaces is not straightforward, especially in three dimensions or at high frequencies when several dispersion surfaces fold back in the Brillouin zone. A recently proposed asymptotic high-frequency homogenization theory is applied to a challenging time-domain experiment with elastic waves in a pinned metallic plate. The prediction of a narrow high-frequency spectral region where the effective medium tensor dramatically switches from positive definite to indefinite is confirmed experimentally; a small frequency shift of the pulse carrier results in two distinct types of highly anisotropic modes. The underlying effective equation mirrors this behavior with a change in form from elliptic to hyperbolic exemplifying the high degree of wave control available and the importance of a simple and effective predictive model.
\end{abstract}

DOI: 10.1103/PhysRevLett.118.254302

The analysis of periodic media underpins advances in electronic properties, wave transport in photonics and acoustics, as well as in interference phenomena throughout many fields of physical and engineering sciences. High symmetry points on dispersion diagrams where the group velocity tends to zero and the density of states is high are often associated with unusual effective properties such as slow light and ultradirectivity $[1,2]$ when the bands display vanishing curvature and even all-angle-negative refraction [3]. More recently, photonics research has focused on Dirac cones following the rise of graphene [4,5] as well as on saddle points in the search for hyperbolic-type features $[6,7]$.

However, identifying the remarkable symmetry points and the nature of their associated mode from a direct reading of the dispersion surfaces is not straightforward. An asymptotic theory that easily identifies the nature of these singularities has been developed [8] that does not require extensive computation of the Bloch dispersion surfaces. Indeed, in contrast to most standard methods such as semianalytic plane-wave $[9,10]$ and multipole expansions [11], numerical finite element or finite difference timedomain methods [12], the so-called high-frequency homogenization (HFH) approach [8] is purely algebraic. Because it only focuses on the apexes of the Brillouin zone, HFH captures the essential features without intensive computation and in an intuitive manner.

The basic methodology has been validated in the microwave regime [13], but for elastic waves, the tensorial equations make any interpretation and prediction extremely difficult, and the situation is far more demanding.
By considering thin plates, the problem becomes more tractable, and several achievements have been reported recently, including broadband cloaking [14,15], negative refraction [16], and lensing [17,18] effects, which parallel the effects observed earlier in electromagnetic metamaterials $[19,20]$. Topical large-scale applications to seismic metamaterials [21] and experiments using pillars [22] or subwavelength resonators attached to elastic plates [23] provide further motivation. The Kirchhoff-Love (KL) plate equation $[24,25]$ provides the simplest model that captures flexural waves, and for structured media such as phononic crystals and metamaterials, the underpinning theory is being advanced accordingly [5,26-28]. The range of validity of KL plate theory, as used for structured media, versus using full elasticity is unclear; for homogeneous plates, the KL theory is only valid for thicknesses up to around $1 / 20$ th of a wavelength [29], and we operate outside of this. Furthermore, the experimental behavior in the time domain and the potential for hyperbolic behavior (which is hard to find experimentally as it is highly dependent upon frequency), all remain unexplored. The full elastic system is awkward to disentangle, and, given its complexity, the simplicity of the KL theory and HFH is appealing; we carefully justify the use of the simplified model in Ref. [30].

In this Letter, the utility of this simplified viewpoint where the elastic wave physics is captured using the simple plate model, and the frequency-dependent anisotropy is identified by the HFH asymptotic approach is demonstrated. We choose to do so for the challenging case of vector elastic waves and this then guides the design of a doubly-periodic 
clamped elastic plate capable of exhibiting the highly directive features of both elliptic and hyperbolic points. Such a constrained elastic plate has a zero-frequency stop band that immediately excludes any possibility of using a quasistatic homogenization method. We show that HFH theory efficiently and accurately models such a system at arbitrarily high frequencies. It predicts the conditions for the observation of a narrow high-frequency spectral region where the effective medium tensor dramatically switches from positive definite to indefinite. Using heterodyne laser interferometry, we measure the time evolution of an elastic Gaussian pulse with central frequency $\Omega_{0}$ within the pinned elastic plate. At the remarkable frequencies $\Omega_{0}$ identified by the HFH theory, we observe highly directive emission and show how a small frequency shift of the pulse carrier results in two distinct types of highly anisotropic modes.

For waves propagating through an infinite periodic structure, one invokes Bloch's theorem [34,35] and considers an elementary cell; the Bloch wave vector $\boldsymbol{\kappa}=$ $\left(\kappa_{1}, \kappa_{2}\right)$ characterizes the phase shift going from one cell to the next, and the dispersion relation connects it with frequency. As is well known in solid state physics [35], only a limited range of wave numbers need normally be considered, namely, the wave numbers along the edges of the irreducible Brillouin zone. The HFH theory focuses on the vicinity of the vertices of the Brillouin zone, which in a square lattice reduce to three high symmetry points $\Gamma, M$, and $X$ located at the apex of a right-angled triangle. Within this framework, the wave field $u$ can be factorized as the product $f(\mathbf{x}) U\left(\mathbf{x} ; \Omega_{0}\right)$ [27] of the Bloch solution $U\left(\mathbf{x} ; \Omega_{0}\right)$ at the vertex at frequency $\Omega_{0}$ and an envelope function $f(\mathbf{x})$ given by the effective equation

$T_{i j}\left(\Omega_{0}\right) \frac{\partial^{2} f}{\partial x_{i} \partial x_{j}}+\left(\Omega_{0}^{2}-\Omega^{2}\right) f=0, \quad(i, j=1,2)$

with $\mathbf{x}=\left(x_{1}, x_{2}\right)$. Equation (1) is a partial differential equation (PDE) of second order which is obtained from an asymptotic analysis of the KL equation which is fourth order; see Refs. [27,30] for the full derivation. $\Omega$ is the wave frequency, and $\Omega_{0}$ is the standing wave frequency at the vertex of the Brillouin zone. In essence, the factorization $f(\mathbf{x}) U\left(\mathbf{x} ; \Omega_{0}\right)$ captures the small-scale variation of the wave field and overcomes the limitation of standard homogenization theories, which consider the wave field uniform at the lattice scale. At the same time, the HFH theory does not require infinite periodic structure and can describe finite-size systems. The nature of the solutions is critically determined by the dynamic effective medium tensor $T_{i j}$. For a lattice cell with reflectional and rotational symmetry (here, a square cell with a circular clamped defect at the center), the off-diagonal terms are zero, and this is just

$$
T_{11}\left(\Omega_{0}\right) \frac{\partial^{2} f}{\partial x_{1}^{2}}+T_{22}\left(\Omega_{0}\right) \frac{\partial^{2} f}{\partial x_{2}^{2}}+\left(\Omega_{0}^{2}-\Omega^{2}\right) f=0 .
$$

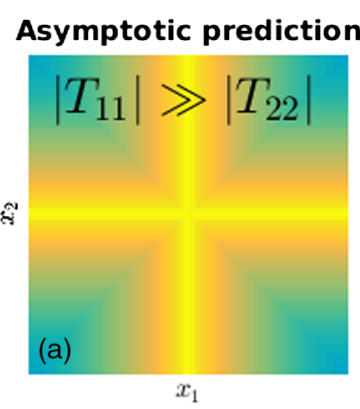

Local Band Structure
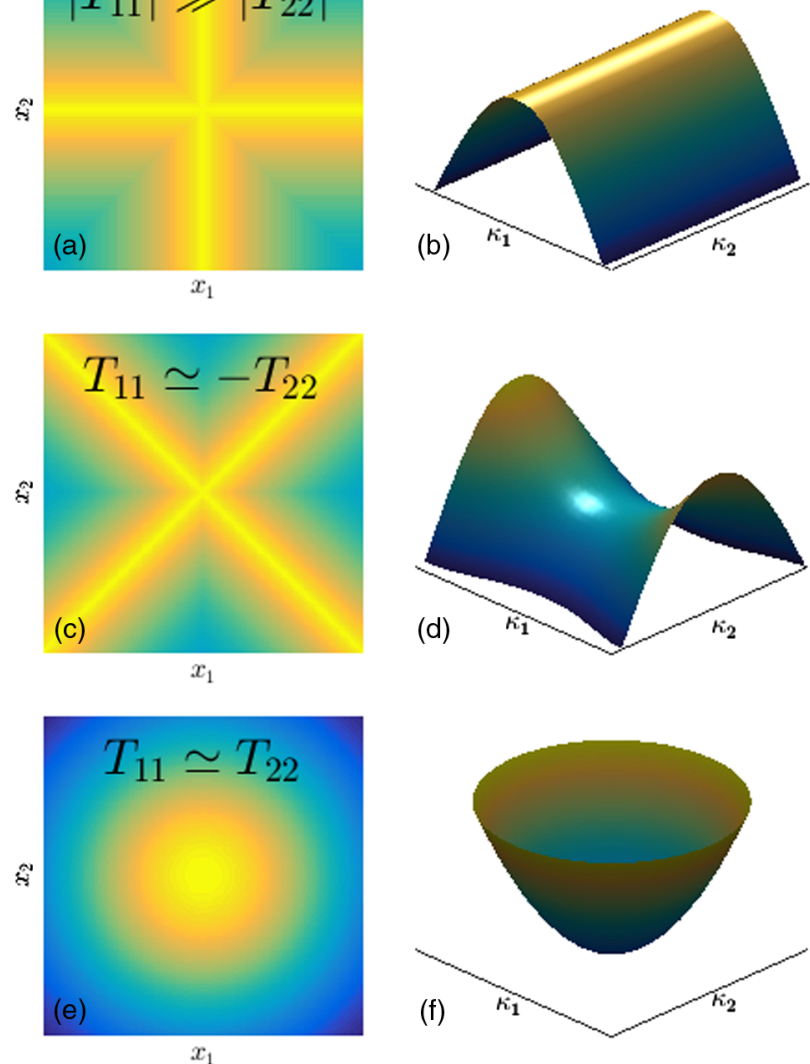

FIG. 1. Asymptotic prediction of the local mode structure and respective dispersion surfaces: Effective medium tensor components govern the parabolic $\left(\left|T_{11}\right| \gg\left|T_{22}\right|\right)$, hyperbolic $\left(T_{11} T_{22}<0\right)$, or elliptic $\left(T_{11} T_{22}>0\right)$ character of Eq. (1) and capture the extreme anisotropic features of waves propagating within a doubly periodic medium shown in (a),(c),(e). The corresponding local dispersion surfaces are shown in (b),(d),(f). The algebraic criteria for critical points straightforwardly extend to $n$-dimensional periodic systems and reveal effective elliptic $\left(T_{11} T_{22}, \ldots, T_{n n}>0\right)$ or hyperbolic $\left(T_{11} T_{22}, \ldots, T_{n n}<0\right)$ features without resorting to visualization of hypersurfaces.

We can immediately see that having both $T_{11}, T_{22}$ of the same sign leads to the PDE in Eq. (2) being of elliptic character; conversely, opposite signs lead to hyperbolic behavior. This change of character is transparently captured by the model and rapidly detected using the HFH theory. Similarly, one can have $T_{11} \gg T_{22}$ or vice versa leading to strong directional behavior along one axis or the other, and this behavior too is predicted. These three types of PDE behaviors, parabolic $\left(\left|T_{11}\right| \gg\left|T_{22}\right|\right)$, hyperbolic $\left(T_{11}=-T_{22}\right)$, and elliptic $\left(T_{11} \simeq T_{22}\right)$, are illustrated in Fig. 1 where the associated modes are shown side by side with the corresponding local dispersion surfaces. The HFH theory, therefore, reduces the difficult problem of interpreting often intricate isofrequency surfaces to the straightforward comparison of the diagonal terms of the tensor, here just two numbers, $T_{i i}$. 


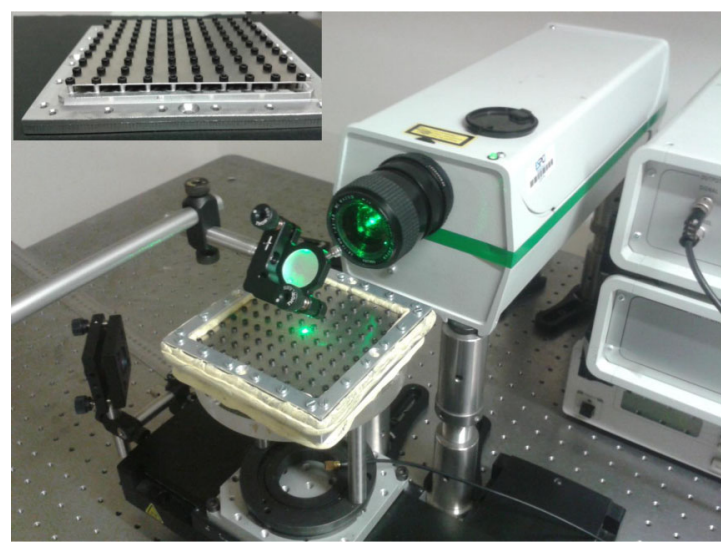

FIG. 2. Experimental apparatus for imaging pinned elastic plate vibrations. The heterodyne laser interferometer (Thalès SH-140) probes the out-of-plane vibration at the surface of a $10 \times 10 \mathrm{~cm}^{2}$ large, 0.5-mm-thick Duralumin plate clamped on a square periodic lattice by 3-mm-diameter columns screwed between the thin plate and a metallic base (see inset). 3-mm-thick adhesive rubber is sandwiched between the plate's edges and a square metallic frame attached to the base to reduce unwanted elastic edge reflections.

Here we want to investigate the predictive capability of the HFH theory in the framework of elastic waves. Therefore, we consider pinned (so-called) platonic crystals (PPCs) created by an array of clamped points, with the objective of achieving highly directive emission of a periodically oscillating source located therein. PPCs are the elastic plate analogue of photonic crystals with infinite conducting rods [36]. Indeed, PPCs display a zero-frequency stop band so that flexural waves with wavelengths arbitrarily long compared to the array pitch cannot propagate.

The system we want to study is shown in Fig. 2. Three mm-diameter and 5-mm-high cylinders have been machined in a Duralumin block to form a $10 \times 10$ square array of supports with 1-cm lattice spacing. Each support has been threaded to enable a $10 \times 10 \mathrm{~cm}^{2} 0.5$-mm-thick vibrating plate $\left(\rho=2789 \mathrm{~kg} / \mathrm{m}^{3}, E=74 \mathrm{GPa}, \nu=0.33\right)$ to be attached securely to it; in the language of elastic plate theory, these are clamped conditions. Although the clamped area is localized to the pin positions, the pins are not precisely pointlike, and so the clamping area is accounted for in the theory.

The KL thin plate theory is often quoted, as in, say, Ref. [29], as only being accurate for wavelengths greater than 20 times the plate thickness, and this condition is not met at the frequencies we consider. However, it is the wavelength in the periodic system that actually matters, and that can be large compared to the plate thickness, even at high frequencies; we justify this technical point further by giving analogous computations for the full vector elastic system in Ref. [30].

Within this thin plate framework, the explicit form of the effective medium tensor $T_{i j}$ for the PPC geometry is given (a)

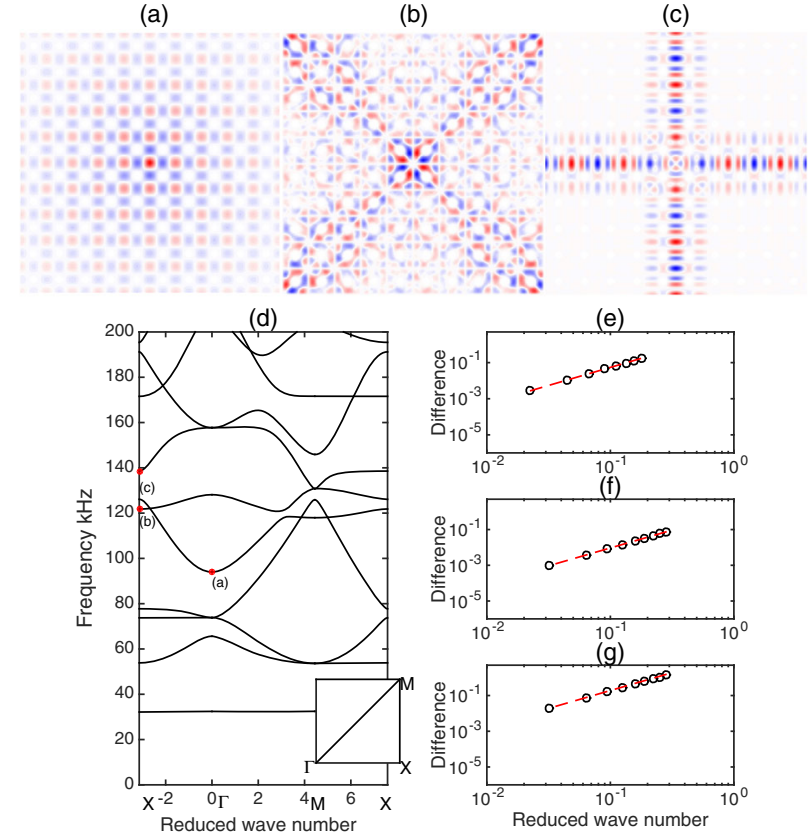

FIG. 3. HFH asymptotics: (a)-(c) The flexural wave field predicted for a point frequency source placed centrally in the array. (a) $94-\mathrm{kHz}$ isotropic response as predicted by effective medium tensor components $T_{11}=T_{22}=1025$; (b) $122-\mathrm{kHz}$ hyperbolic behavior following from $T_{11}=-180.17$ and $T_{22}=311.74$; (c) $138 \mathrm{kHz}$ creating a + shape with $T_{11}=$ -4189.10 and $T_{22}=19.51$. (d) Bloch dispersion diagram using the irreducible Brillouin zone ГXM (shown in inset) with frequencies used in (a)-(c) as red dots. (e)-(g) Log-log validates the HFH asymptotics in the neighborhood of the frequencies used in (a)-(c). Circles are HFH asymptotics of the dispersion curves for the $X \Gamma$ path; dashed curves are from finite element numerics for the KL model, and the vertical scale is the difference of frequency from that at the band edge.

in Ref. [27]. The HFH theory is able to discriminate between interesting and uninteresting symmetry points and capture a rich array of behaviors for the given experimental parameters. In our case, we select three frequencies of interest at which modes with remarkable properties are predicted. At $122 \mathrm{kHz}$ near symmetry point $X$, the coefficients $T_{i i}$ in the effective tensor of the HFH theory have opposite sign, $T_{11}=-180.172$ and $T_{22}=311.7432$, and are close enough to reflect hyperbolic behavior analogue to Fig. 1(b). For excitation at the center of the system, the predicted theoretical wave field distribution at this particular frequency is shown in Fig. 3(b): The mode is highly directive and is aligned along the diagonals of the system. At $138 \mathrm{kHz}$, strongly differing $T_{i i}$ coefficients predict another very distinct anisotropy within the effective material [Fig. 3(c)], $\left|T_{22} / T_{11}\right| \sim 0.005$, leading to the effective equation being parabolic. The mode is expected now to radiate along the lattice axes as shown in Fig. 1(a). For completeness, we discuss the situation of equal $T_{11}, T_{22}$ as shown in Fig. 1(c); here we expect isotropy and elliptic effective equations. The HFH prediction extends 
beyond the symmetry points with a dispersion relation local to the band edge given in terms of the effective tensor

$$
\Omega \sim \Omega_{0}-\frac{T_{i j}\left(\Omega_{0}\right)}{2 \Omega_{0}} \kappa_{i} \kappa_{j},
$$

where $\kappa_{i}=K_{i}-d_{i}$ and $d_{i}=0,-\pi, \pi$ depending on the band edge chosen.

In the experiment, the ultrasound emission is produced by a broadband piezoelectric transducer (Panametrics M109). A silica needle is stuck on the transducer and put in contact with the bottom surface of the plate. We generate a single-oscillation short pulse excitation synthesized by an arbitrary waveform generator (Agilent 33120A). The out-of-plane displacement is measured point by point with a broadband heterodyne interferometric laser probe (Thalès SH140). The probe is scanned on a square grid with 0.68 -mm step resolution, and the spatiotemporal distribution of the vibration field at the surface of the plate is reconstructed. The time signal for each point is averaged 10 times as a compromise to eliminate most of the noise and yet conserve a reasonable measurement time. The data are subsequently filtered to obtain the elastic response to a narrow bandwidth $\left(\Delta \Omega_{0}\right)$ Gaussian pulse with central frequency $\Omega_{0}$ easily adjustable around theoretical prediction. To reduce elastic reflections at the edges of the plate and mimic a finite array, surrounded by perfectly matched layers, a 3-mm-thick layer of adhesive rubber is sandwiched between the edges of the vibrating plate and a rigid metallic frame (see Fig. 2). This method efficiently absorbs elastic waves in the $\mathrm{kHz}$ range and reduces unwanted reflections [18]; we measured an attenuation of $8 \mathrm{~dB}$ after reflection from the absorbing boundaries. The experimental setup is shown in Fig. 2.

The experimental results are presented in Fig. 4, together with time-domain simulations of the pinned plate using the SIMSONIC package [37] which solves the full 3D Navier system with the finite difference time-domain (FDTD) method with absorbing boundary conditions. In contrast to the modal representation shown in Fig. 1, here we show snapshots of the time evolution of the elastic field. The complete temporal buildup of this mode is shown as a movie in Ref. [30]. Nevertheless, the hyperbolic and elliptic behavior are well captured with highly directional radiation at frequencies close to the prediction. Figures 4(c) and 4(d) show the $X$ shape obtained at $\Omega_{0}=105 \mathrm{kHz}$ $\left(\Delta \Omega_{0}=10 \mathrm{kHz}\right)$, which is characteristic of hyperbolic dispersion. This contrasts with the + shape emission obtained at $\Omega_{0}=128 \mathrm{kHz}\left(\Delta \Omega_{0}=10 \mathrm{kHz}\right)$ and shown in Figs. 4(e) and 4(f). The elliptic isotropic behavior is at $\Omega_{0}=90 \mathrm{kHz}\left(\Delta \Omega_{0}=10 \mathrm{kHz}\right)$ as in Figs. 4(a) and 4(b). Resonant frequencies found numerically $(95,110$, and $119 \mathrm{kHz}$ ) differ from the experimental frequencies by less than $10 \%$. The small difference can be attributed to the absence of residual reflections in the simulations where perfectly absorbing boundaries allow full establishment of (a)

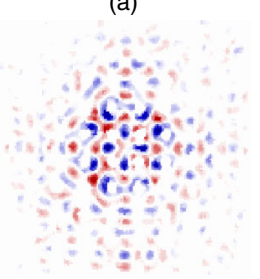

(c)

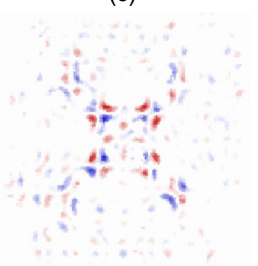

(e)

(b)

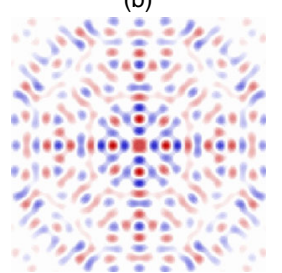

(d)

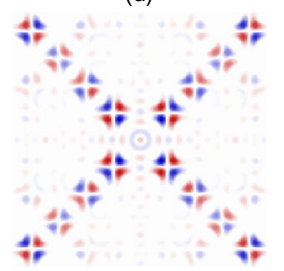

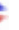

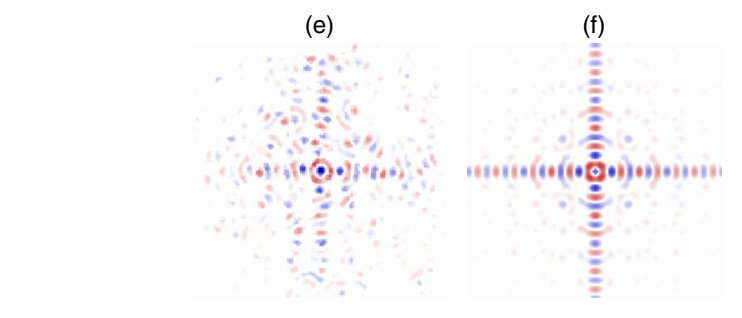

FIG. 4. Snapshots of flexural wave field evolution from a pulse excitation at the center of the system with central frequency $\Omega$ and bandwidth $\Delta \Omega$. (a),(c),(e) Experiment at $\Omega=90,105,128 \mathrm{kHz}$ and $\Delta \Omega=10 \mathrm{kHz}$; see, also, the video in Ref. [30]. We observe wavelengths at $90 \mathrm{kHz}: 9 \mathrm{~mm}$, at $105 \mathrm{kHz}: 27.9 \mathrm{~mm}$, and at $128 \mathrm{kHz}: 6.8 \mathrm{~mm}$, which are at least 10 times the scanning resolution. (b),(d),(f) Numerical simulations, FDTD solving the full 3D Navier system, at $\Omega=95,110,119 \mathrm{kHz}$ with $\Delta \Omega=1 \mathrm{kHz}$. The minor difference in frequency is attributed to finite boundary effects in the experiments.

the modes. Note also that the numerical distributions are sharper, as the bandwidth used in the simulations is only $\Delta \Omega_{0}=1 \mathrm{kHz}$, leading to better spectral selectivity. Actually, the choice of the pulse bandwidth in the experiment was crucial and delicate, as a trade-off was to be found between sharp modal selectivity (narrow bandwidth) and early establishment of the mode (short pulse) before edges are reached and reflections perturb the mode distribution. This problem is less of an issue in the numerics, although $\Delta \Omega_{0}$ is kept reasonably large to limit the mode establishment time and computational time. As a result, a residual trace of the + shaped mode is seen on top of the $X$-shaped mode, e.g., in Fig. 4(d). The minor frequency offset between experiment and theory is also an expected consequence of modeling the plate vibration with KL plate theory; this offset being also seen in the detailed comparisons with the full elastic dispersion diagram of Ref. [30].

In this Letter, the recently introduced that $\mathrm{HFH}$ was applied to the KL thin plate model, in a counterintuitive high-frequency limit where it might conventionally not be expected to hold, to predict critical frequencies at which striking, strong anisotropic wave patterns are generated within a periodically pinned plate. Experimental 
observations confirmed these asymptotic predictions, as did time-domain computations.

We were drawn to this more intuitive, asymptotic approach as the available alternatives require heavy computational resources and, at high frequencies, produce results hard to interpret as illustrated in Ref. [30]. These issues were shared with many other complex doubly and triply periodic media, such as photonic and phononic crystals. The techniques developed and illustrated herein are also valuable in these contexts.

Potential applications are for slow waves and waveguiding in delay lines (one can achieve " $U$-turns" in PPCs without line defects, as shown in Ref. [30]), superlensing [6] and most importantly to energy harvesting through the Purcell effect; the latter taking advantage of the high local density of states at the critical frequencies [6,38,39]. Notably a pristine plate could be used and mimic a periodically pinned plate by making use of an array of magnets or alternatively of electrodes deposited on top and bottom surfaces of a piezoelectric plate.

R. V.C. thanks the Engineering and Physical Sciences Research Council (EPSRC) (UK) for support. S. G. is thankful for an European Research Council (ERC) starting grant (ANAMORPHISM). P. S. is thankful to the Agence Nationale de la Recherche support under grant ANR PLATON (Grant No. 12-BS09-003-01), the LABEX WIFI (Laboratory of Excellence within the French Program Investments for the Future) under References Nos. ANR-10-LABX-24 and ANR-10-IDEX-0001-02 PSL*, and the PICS-ALAMO. This research was supported in part by The Israel Science Foundation (Grants No. 1871/ 15 and 2074/15) and the United States-Israel Binational Science Foundation NSF/BSF (Grant No. 2015694).

* Corresponding author. patrick.sebbah@espci.fr

[1] K. Sakoda, Optical Properties of Photonic Crystals (Springer-Verlag, Berlin, 2001).

[2] B. Gralak, S. Enoch, and G. Tayeb, J. Opt. Soc. Am. A 17, 1012 (2000).

[3] C. Luo, S. G. Johnson, J. D. Joannopoulos, and J. B. Pendry, Phys. Rev. B 65, 201104 (2002).

[4] A. H. C. Neto, H. F. Guinea, N. M. R. Peres, K. S. Novoselov, and A. K. Geim, Rev. Mod. Phys. 81, 109 (2009).

[5] D. Torrent, D. Mayou, and J. Sánchez-Dehesa, Phys. Rev. B 87, 115143 (2013).

[6] A. Poddubny, I. Iorsh, P. Belov, and Y. Kivshar, Nat. Photonics 7, 948 (2013).

[7] V. P. Drachev, V.A. Podolskly, and A. V. Kildishev, Opt. Express 21, 15048 (2013).

[8] R. V. Craster, J. Kaplunov, and A. V. Pichugin, Proc. R. Soc. A 466, 2341 (2010).

[9] K. M. Ho, C. T. Chan, and C. M. Soukoulis, Phys. Rev. Lett. 65, 3152 (1990).
[10] S. G. Johnson and J. D. Joannopoulos, Opt. Express 8, 173 (2001).

[11] C. G. Poulton, A. B. Movchan, R. C. McPhedran, N. A. Nicorovici, and Y. A. Antipov, Proc. R. Soc. A 456, 2543 (2000).

[12] F. Zolla, G. Renversez, A. Nicolet, B. Kuhlmey, S. Guenneau, and D. Felbacq, Foundations of Photonic Crystal Fibres (Imperial College Press, London, 2005).

[13] L. Ceresoli, R. Abdeddaim, T. Antonakakis, B. Maling, M. Chmiaa, P. Sabouroux, G. Tayeb, S. Enoch, R. V. Craster, and S. Guenneau, Phys. Rev. B 92, 174307 (2015).

[14] M. Farhat, S. Guenneau, and S. Enoch, Phys. Rev. Lett. 103, 024301 (2009).

[15] N. Stenger, M. Wilhelm, and M. Wegener, Phys. Rev. Lett. 108, 014301 (2012).

[16] B. Bonello, L. L. Belliard, J. Pierre, J. O. Vasseur, B. Perrin, and O. Boyko, Phys. Rev. B 82, 104109 (2010).

[17] M. Dubois, M. Farhat, E. Bossy, S. Enoch, S. Guenneau, and P. Sebbah, Appl. Phys. Lett. 103, 071915 (2013).

[18] G. Lefebvre, M. Dubois, R. Beauvais, Y. Achaoui, R. K. Ing, S. Guenneau, and P. Sebbah, Appl. Phys. Lett. 106, 024101 (2015).

[19] D. R. Smith, J. B. Pendry, and M. C. K. Wiltshire, Science 305, 788 (2004).

[20] S. A. Ramakrishna, Rep. Prog. Phys. 68, 449 (2005).

[21] S. Brule, E. H. Javelaud, S. Enoch, and S. Guenneau, Phys. Rev. Lett. 112, 133901 (2014).

[22] Y. Pennec, B. Djafari-Rouhani, H. Larabi, J. O. Vasseur, and A. C. Hladky-Hennion, Phys. Rev. B 78, 104105 (2008).

[23] A. Colombi, P. Roux, and M. Rupin, J. Acoust. Soc. Am. 136, EL192 (2014).

[24] L. D. Landau and E. M. Lifshitz, Theory of Elasticity, 2nd ed. (Pergamon Press, New York, 1970).

[25] K. F. Graff, Wave Motion in Elastic Solids (Oxford University Press, New York, 1975).

[26] A. B. Movchan, N. V. Movchan, and R. C. McPhedran, Proc. R. Soc. A 463, 2505 (2007).

[27] T. Antonakakis and R. V. Craster, Proc. R. Soc. A 468, 1408 (2012).

[28] R. C. McPhedran, A. B. Movchan, N. V. Movchan, M. Brun, and M. J. A. Smith, Proc. R. Soc. A 471, 20140746 (2015).

[29] L. R. F. Rose and C. H. Wang, J. Acoust. Soc. Am. 116, 154 (2004).

[30] See the Supplemental Material at http://link.aps.org/ supplemental/10.1103/PhysRevLett.118.254302, which includes Refs. [31-33], for the full 3D computation of the Navier system of equations of elastodynamics for the periodically pinned plate and the isofrequency contours near the symmetry points, and for the use of the wave guiding effect near a symmetry point to bend the wave around a $U$-shape trajectory. Included are links to a movie which shows the experimental temporal buildup of the mode of Fig. 4(c) and a video of the simulation of the bouncing energy following a $U$-shape trajectory using dynamic anisotropy.

[31] R. V. Craster and S. Guenneau, Acoustic Metamaterials (Springer Verlag, London, 2012).

[32] A. Diatta and S. Guenneau, Appl. Phys. Lett. 105, 021901 (2014). 
[33] D. R. Smith and D. Schurig, Phys. Rev. Lett. 90, 077405 (2003).

[34] C. Kittel, Introduction to Solid State Physics, 7th ed. (John Wiley \& Sons, New York, 1996).

[35] L. Brillouin, Wave Propagation in Periodic Structures, 2nd ed. (Dover, New York, 1953).

[36] N. A. Nicorovici, R. C. McPhedran, and L. C. Botten, Phys. Rev. Lett. 75, 1507 (1995).
[37] E. Bossy, M. Talmant, and P. Laugier, J. Acoust. Soc. Am. 115, 2314 (2004).

[38] A. P. Slobozhanyuk, P. Ginzburg, D. A. Powell, I. Iorsh, A. S. Shalin, P. Segovia, A. V. Krasavin, G. A. Wurtz, V. A. Podolskiy, P. A. Belov, and A. V. Zayats, Phys. Rev. B 92, 195127 (2015).

[39] K. Rustomji, R. Abdeddaim, C. M. de Sterke, B. Kuhlmey, and S. Enoch, Phys. Rev. B 95, 035156 (2017). 